\title{
Author Correction: Mapping the proximity interaction network of the Rho-family GTPases reveals signalling pathways and regulatory mechanisms
}

Halil Bagci (D), Neera Sriskandarajah, Amélie Robert, Jonathan Boulais (D), Islam E. Elkholi, Viviane Tran, Zhen-Yuan Lin, Marie-Pier Thibault, Nadia Dubé, Denis Faubert, David R. Hipfner, Anne-Claude Gingras (DD and Jean-François Côté (D)

${ }^{9}$ These authors contributed equally: David R. Hipfner, Anne-Claude Gingras.

Correction to: Nature Cell Biology https://doi.org/10.1038/s41556-019-0438-7, published online 23 December 2019.

In the version of this article originally published, incorrect versions of Supplementary Tables 7 and 8 were included in the Supplementary Table file. The error has been corrected in the HTML version of the paper.

Published online: 10 February 2020

https://doi.org/10.1038/s41556-020-0479-y

๑ The Author(s), under exclusive licence to Springer Nature Limited 2020

\section{Author Correction: Ribosomes guide pachytene piRNA formation on long intergenic piRNA precursors}

Yu H. Sun (D), Jiang Zhu, Li Huitong Xie, Ziwei Li, Rajyalakshmi Meduri, Xiaopeng Zhu, Chi Song (iD, Chen Chen,

Emiliano P. Ricci, Zhiping Weng and Xin Zhiguo Li (D)

Correction to Nature Cell Biology https://doi.org/10.1038/s41556-019-0457-4, published online 3 February 2020

In the version of this article originally published, there was a typographical error in the legend for Fig. 8a. In "for-dual strand clusters, blue represents Walston strand mapping reads and red represents Crick strand mapping reads,' 'Walston' should be 'Watson'. The error has been corrected in the HTML and PDF versions of the paper.

Published online: 17 February 2020

https://doi.org/10.1038/s41556-020-0482-3

(C) The Author(s), under exclusive licence to Springer Nature Limited 2020 